\title{
Dihydroartemisinin increases apoptosis of colon cancer cells through targeting Janus kinase 2/signal transducer and activator of transcription 3 signaling
}

\author{
DONGSHENG WANG ${ }^{1}$, BEI ZHONG ${ }^{2}, \mathrm{YU} \mathrm{LI}^{1}$ and XIAODONG LIU ${ }^{1}$ \\ Departments of ${ }^{1}$ General Surgery and ${ }^{2}$ Hyperbaric Oxygen, \\ The Affiliated Hospital of Qingdao University, Qingdao, Shandong 266003, P.R. China
}

Received September 30, 2016; Accepted September 1, 2017

DOI: $10.3892 / \mathrm{ol} .2017 .7502$

\begin{abstract}
As a derivative of artemisinin, dihydroartemisinin is effective in the treatment of malaria. Dihydroartemisinin has been identified to possess inhibitory effects in numerous types of animal model with tumors, indicating that it has an antineoplastic effect. The aim of the present study was to analyze the potential anticancer effects of dihydroartemisinin, particularly its effect on apoptosis of colon cancer cells. In the present study, it was identified that dihydroartemisinin inhibited cell viability, promoted cell apoptosis, increased B-cell lymphoma-2-associated $\mathrm{X}$-protein expression, increased caspase-3/9 activities, decreased poly(ADP-ribose) polymerase levels, decreased phosphorylation of extracellular-signal-regulated kinase, and increased phosphorylation of c-Jun N-terminal kinase and p38 mitogen-activated protein kinase in colon cancer cells. Conversely, the phosphorylation of Janus kinase 2 (JAK2) and signal transducer and activator of transcription 3 (STAT3) was suppressed by dihydroartemisinin in colon cancer cells. These results demonstrate that the potential anticancer effects of dihydroartemisinin may increase apoptosis of colon cancer cells through targeting JAK2/STAT3 signaling.
\end{abstract}

\section{Introduction}

Colorectal cancer (CRC) is a common malignant tumor. With increasing life expectancy and ongoing changes in diet, the incidence rate of CRC is increasing each year in China (1). Although early screening has decreased the mortality rate of patients with CRC, numerous cases are not diagnosed until the disease has progressed to the middle and late stages (2).

Correspondence to: Dr Dongsheng Wang, Department of General Surgery, The Affiliated Hospital of Qingdao University, 16 Jiangsu Road, Qingdao, Shandong 266003, P.R. China

E-mail: fwel6684659@126.com

Key words: dihydroartemisinin, colon cancer, apoptosis, Janus kinase 2, signal transducer and activator of transcription 3
Additionally, although improvements in the diagnosis and treatment of CRC have increased the 5-year survival rate of patients to a certain extent, the proliferation and metastasis of tumors still present challenges in the treatment of CRC (3). In order to suppress the progression of CRC, inhibition of tumor metastasis, prevention of tumor spreading and recurrence, targeted interventions combined with traditional surgery, chemotherapy and radiotherapy are expected to be important tools to increase the survival rate of patients with CRC $(4,5)$.

Signal transducer and activator of transcription (STAT) 3 , a member of the STAT protein family, may be activated through phosphorylation by a variety of extracellular growth factors and inflammatory mediators (6). Constitutively activated [phosphorylated (p-)STAT3] was identified in numerous tumor cell lines and a number of human tumors. p-STAT3 is significantly associated with tumor invasion and poor prognosis of CRC (6). p-STAT3 exhibits marked expression levels in pancreatic cancer and is associated with tumor staging and metastasis to lymph nodes (6). Janus kinase 2 (JAK2) is associated with the embryonic development of normal individuals and is widely expressed in various types of cell, catalyzing the immune responses induced by cytokines (7). Through the change in cytokine levels and expression levels of cell membrane receptors, activated JAK2/STAT3 serves a role in two-way regulation of immune response and inflammation, and therefore may be able to enhance or inhibit an immune response (7). The activation of STAT3 serves an important function in tumor progression, particularly in tumors associated with chronic inflammation, including inflammatory colitis-associated CRC, liver cancer and pancreatic cancer (8). Sustained activation of JAK2/STAT3 is associated with tumorigenesis, differentiation, proliferation, apoptosis, angiogenesis, recruitment of immune cells and metastasis of tumors (9).

Artemisinin is a sesquiterpene lactone composed of endoperoxides, which is derived from the plant Artemisia annua $\mathrm{L}$. (Fig. 1). Artemisinin and its derivatives possess a wide range of pharmacological properties, including anti-Plasmodium, anti-schistosomiasis, antiviral and antitumor effects. As a result, artemisinin derivatives have been successfully developed into antimalarial drugs (10). In recent years, the antineoplastic effect of artemisinin and its derivatives has 
become a topic of research. We previously investigated and reported on the antineoplastic effect and underlying molecular mechanism of such compounds (11). The antineoplastic mechanism of dihydroartemisinin may be the result of its inhibitory effect on the proliferation of tumor cells through the induction of cell cycle arrest and promotion of apoptosis, as well as inhibition of angiogenesis, invasion and metastasis of tumor cells (12). Intracellular ferrous sulfate and heme may be the targets of dihydroartemisinin, resulting in inhibition of tumor cells (12). Since dihydroartemisinin exhibits a number of advantages including broad-spectrum antitumor effects and low toxicity to normal cells, in addition to its high efficacy in killing tumor cells with multiple drug resistance, it is possible that it may be developed into a new antitumor drug $(10,13)$. In the present study, the effect of dihydroartemisinin treatment on viability, apoptosis, JAK2 levels and STAT3 levels in HCT116 cells was investigated. In addition, the effect of dihydroartemisinin treatment on levels of B-cell lymphoma-2-associated X-protein (BAX), caspase-3 and caspase-9 (caspase-3/9), poly (ADP-ribose) polymerase (PARP), p38 mitogen-activated protein kinase (p38 MAPK), c-Jun N-terminal kinases $1 / 2(\mathrm{JNK} 1 / 2)$ and extracellular-signal-related kinases $1 / 2$ (ERK1/2) in HCT116 cells was investigated in order to elucidate the underlying molecular mechanism of dihydroartemisinin-induced apoptosis.

\section{Materials and methods}

Cell culture and treatments. Human HCT116 CRC cells were purchased from the Shanghai Cell Bank of the Chinese Academy of Sciences (Shanghai, China) and cultured in RPMI-1640 medium (GE Healthcare Life Sciences, Logan, UT, USA), with $10 \%$ fetal bovine serum (Thermo Fisher Scientific, Inc., Waltham, MA, USA), 2 mM L-glutamine (Merck KGaA, Darmstadt, Germany), $100 \mathrm{U} / \mathrm{ml}$ penicillin (Merck KGaA) and $100 \mu \mathrm{g} / \mathrm{ml}$ streptomycin (Merck KGaA) at $37^{\circ} \mathrm{C}$ in a humidified atmosphere containing $5 \% \mathrm{CO}_{2}$. Dihydroartemisinin was purchased from Merck $\mathrm{KGaA}$ and its structural formula is presented in Fig. 1.

Cell viability assay. Cells were plated at $4 \times 10^{3}$ cells/well in 96-well plates and treated with $0,12.5,25,50$ and $100 \mu \mathrm{M}$ dihydroartemisinin for 24,48 and $72 \mathrm{~h}$. MTT assay reagent ( $5 \mathrm{mg} / \mathrm{ml}$; Beyotime Institute of Biotechnology, Haimen, China) was added to each well prior to incubation for $4 \mathrm{~h}$ at $37^{\circ} \mathrm{C}$. The culture medium was removed from the wells and $150 \mu \mathrm{l} /$ well dimethylsulfoxide was added to each well for $10 \mathrm{~min}$. Absorbance was measured using a Victor Light system (PerkinElmer, Waltham, MA, USA) at $490 \mathrm{~nm}$ with an enzyme-linked immunosorbent detector (Model 550; Bio-Rad Laboratories, Inc., Hercules, CA, USA).

Flow cytometry. Cells were plated at $1 \times 10^{5}$ cells/well in 6-well plates and treated with 25,50 and $100 \mu \mathrm{M}$ dihydroartemisinin for $48 \mathrm{~h}$. Cells were harvested using centrifugation at 1,000 x g for $5 \mathrm{~min}$ at $4^{\circ} \mathrm{C}$ and resuspended in binding buffer (BD Biosciences, San Jose, CA, USA). Cells were incubated with Annexin V-fluorescein isothiocyanate and propidium iodide in the dark for $15 \mathrm{~min}$ at room temperature. Cell apoptosis was analyzed using cytometry using a FACSCalibur flow cytometer (BD Biosciences) and calculated using WinMDI 2.9 software (The Scripps Research Institute, La Jolla, CA, USA).

Caspase-3/9 activity assay. Cells were $\left(1 \times 10^{6}\right.$ cell/well $)$ harvested using centrifugation at $1,000 \mathrm{x} \mathrm{g}$ for $5 \mathrm{~min}$ at $4^{\circ} \mathrm{C}$ and washed with ice-cold PBS. Cells were lysed in radioimmunoprecipitation (RIPA) assay buffer (Beyotime Institute of Biotechnology) on ice for $10 \mathrm{~min}$ and protein concentrations were determined using a bicinchoninic acid assay (Beyotime Institute of Biotechnology). Equal amounts of protein $(10 \mu \mathrm{g})$ were incubated with caspase-3/9 activity kits (Beyotime Institute of Biotechnology) at $37^{\circ} \mathrm{C}$ for $1 \mathrm{~h}$ and the absorbance was measured using an enzyme-linked immunosorbent detector at $405 \mathrm{~nm}$.

Western blotting. Cells were harvested after 1,000 x $\mathrm{g}$ for 5 min at $4^{\circ} \mathrm{C}$ and washed with ice-cold PBS. Cells were lysed in RIPA assay buffer (Beyotime Institute of Biotechnology) on ice for $10 \mathrm{~min}$ and protein concentrations were determined using a bicinchoninic acid assay. Equal amounts of protein $(50 \mu \mathrm{g})$ were separated by SDS-PAGE (8-10\% gel) and transferred onto a polyvinylidene fluoride membrane (Bio-Rad Laboratories, Inc.). Following blocking with skimmed milk (5\%) suspended in tris-buffered saline with Tween 20 overnight at $4^{\circ} \mathrm{C}$, membranes were incubated with the following primary antibodies: Anti-BAX (cat no. 5023; 1:2,000; Cell Signaling Technology, Inc., Danvers, MA, USA), anti-PARP (cat no. 9532; 1:2,000; Cell Signaling Technology, Inc.), anti-phosphorylated ERK1/2 (cat no. 8544; 1:1,000; Cell Signaling Technology, Inc.), anti-phosphorylated JNK1/2 (cat no. 9255; 1:1,000; Cell Signaling Technology, Inc.), anti-phosphorylated p38 MAPK (cat no. 4511; 1:1,000; Cell Signaling Technology, Inc.) and GAPDH (cat no. 5174; 1:2,000; Cell Signaling Technology, Inc.) overnight at $4^{\circ} \mathrm{C}$. Following washing, membranes were probed with horseradish peroxidase-conjugated secondary antibodies (cat no. 14708; 1:5,000; Cell Signaling Technology, Inc.). Protein blank was visualized using a chemiluminescence kit (Thermo Fisher Scientific, Inc.).

Statistical analyses. Results are expressed as the mean \pm standard deviation. Comparison of multiple experimental groups was performed by one-way analysis of variance followed by a post hoc Bonferroni modification of multiple comparison t-tests. $\mathrm{P}<0.05$ was considered to indicate a statistically significant difference.

\section{Results}

Effects of dihydroartemisinin on the viability of CRC cells. The anticancer effects of dihydroartemisinin on the viability of CRC cells were revealed using an MTT assay. As presented in Fig. 2, treatment with dihydroartemisinin decreased the viability of HCT116 cells in a dose- and time-dependent manner. In particular, treatment with dihydroartemisinin significantly decreased the viability of HCT116 cells after 24, 48 and $72 \mathrm{~h}$.

Effects of dihydroartemisinin on the apoptosis of CRC cells. The anticancer effects of dihydroartemisinin on the apoptosis of CRC cells were investigated using flow cytometry. 


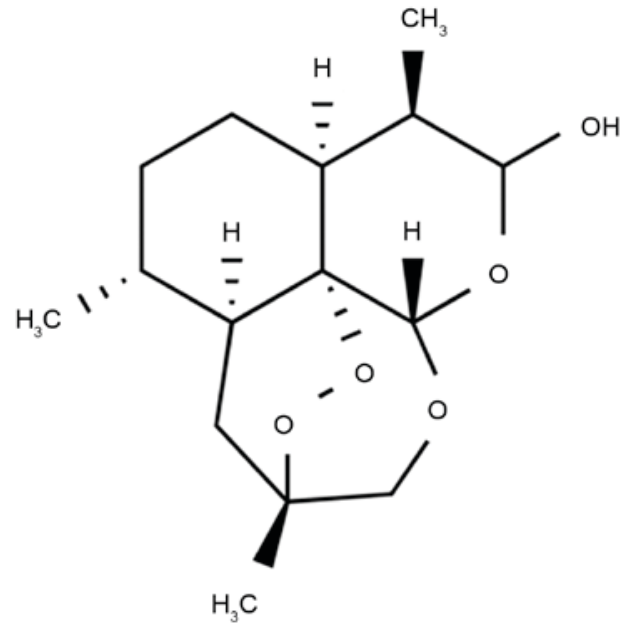

Figure 1. Chemical structure of dihydroartemisinin.

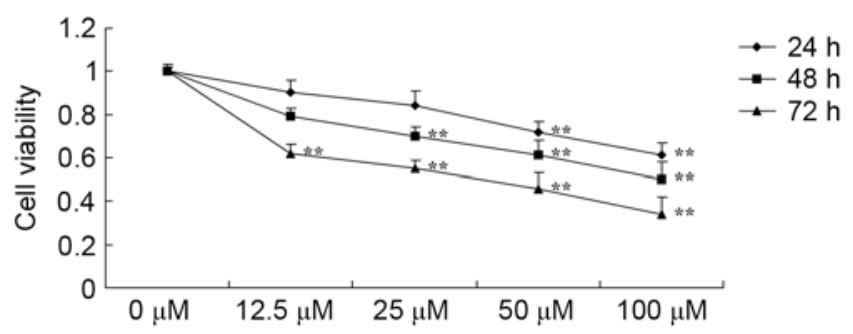

Figure 2. Effect of dihydroartemisinin on the viability of colon cancer cells. ${ }^{* *} \mathrm{P}<0.01$ compared with the $0 \mu \mathrm{M}$ dihydroartemisinin group.

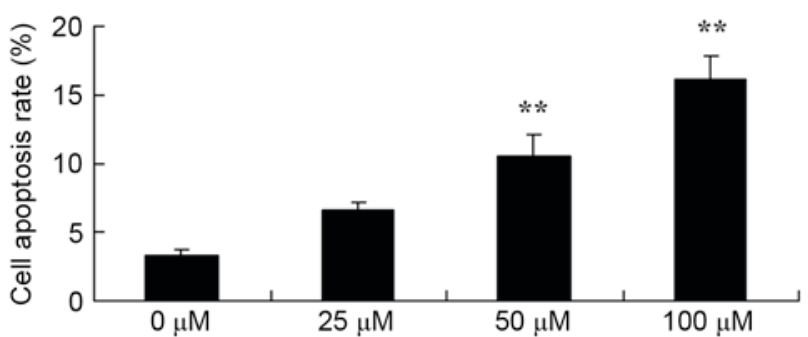

Figure 3. Effect of dihydroartemisinin on apoptosis of colon cancer cells ${ }^{* *} \mathrm{P}<0.01$ compared with the $0 \mu \mathrm{M}$ dihydroartemisinin group.

As presented in Fig. 3, treatment with dihydroartemisinin significantly increased the apoptotic rate of HCT116 cells in a dose-dependent manner over a 48 -h period.

Effects of dihydroartemisinin on BAX and PARP expression in $C R C$ cells. To investigate the effect of dihydroartemisinin on BAX and PARP expression in CRC cells, BAX protein expression was evaluated using western blotting. As presented in Fig. 4, treatment with dihydroartemisinin significantly increased BAX expression and significantly decreased PARP protein expression in HCT116 cells in a dose-dependent manner over a 48 -h period.

Effect of dihydroartemisinin on caspase-3/9 activity in CRC cells. In the present study, it was confirmed that dihydroartemisinin had an effect on caspase-3/9 activity in CRC cells.
As presented in Fig. 5, there was a significant dose-dependent increase in caspase-3/9 activity in CRC cells as a result of treatment with dihydroartemisinin $(50$ and $100 \mu \mathrm{M})$ over a 48-h period.

Effects of dihydroartemisinin on phosphorylation of ERK1/2, $J N K 1 / 2$ and 38 MAPK in CRC cells. To determine the endogenous function of MAPK in the effects of dihydroartemisinin on CRC cells, the phosphorylation of ERK1/2, JNK1/2 and p38 MAPK was analyzed. As presented in Fig. 6, dihydroartemisinin significantly suppressed the phosphorylation of ERK1/2, and induced the phosphorylation of JNK1/2 and p38 MAPK in HCT116 cells in a dose-dependent manner over a 48-h period.

Effects of dihydroartemisinin on phosphorylation of JAK2 and STAT3 protein expression in CRC cells. The role of the JAK and STAT3 signaling pathway in the death of CRC cells by dihydroartemisinin was further verified using western blotting. As presented in Fig. 7, dihydroartemisinin significantly suppressed the phosphorylation of JAK2 and STAT3 in CRC cells in a dose-dependent manner over a 48 -h period.

\section{Discussion}

CRC is one of the most common malignant tumors globally. With an annual incidence of $<940,000$ novel cases, almost 500,000 succumb to CRC annually (14). Among the numerous types of digestive tract cancer, CRC has the third highest incidence rate in China, second only to gastric cancer and esophageal cancer. With the increase in life expectancy of the general population, the annual incidence rate of CRC has increased by $3.9 \%$ in China since 2005, surpassing the world average of $2 \%$ (15). Although early screening, early diagnosis and early treatment are desirable in the treatment of CRC, numerous cases are not detected until the middle and late stages, when the risk of tumor metastasis and spreading is increased (8). The incidence and development of the majority of malignant tumors is associated with the inhibition of tumor cell apoptosis and evasion of immune surveillance (5). The results of the present study suggest that dihydroartemisinin decreased cell viability, induced apoptosis, increased caspase-3/9 activity and upregulated BAX protein expression in HCT116 cells.

PARP, a monomer protein with a zinc-finger structure, is widely distributed in eukaryotic cells in a non-active form, and a single-stranded DNA break (SSB) or free DNA terminal is the only factor that is able to activate the enzyme, whereas NAD ${ }^{+}$ is its only substrate (16). PARP may be activated following recognition and binding to an SSB or a free end of DNA, whereby it catalyzes the synthesis of poly(ADP-ribose) (17). In so doing, the enzyme modifies numerous nuclear proteins and enzymes following translation, thus regulating a series of molecular events in cells. As PARP is a protease with numerous important functions, it is associated with the incidence and development of a number of diseases (18). The results of the present study suggest that treatment with dihydroartemisinin significantly suppressed PARP levels in HCT116 cell. Wang et al (19) reported that dihydroartemisinin inhibits the growth and induces autophagy of iron-loaded human myeloid leukemia via PARP expression. These results suggest that 


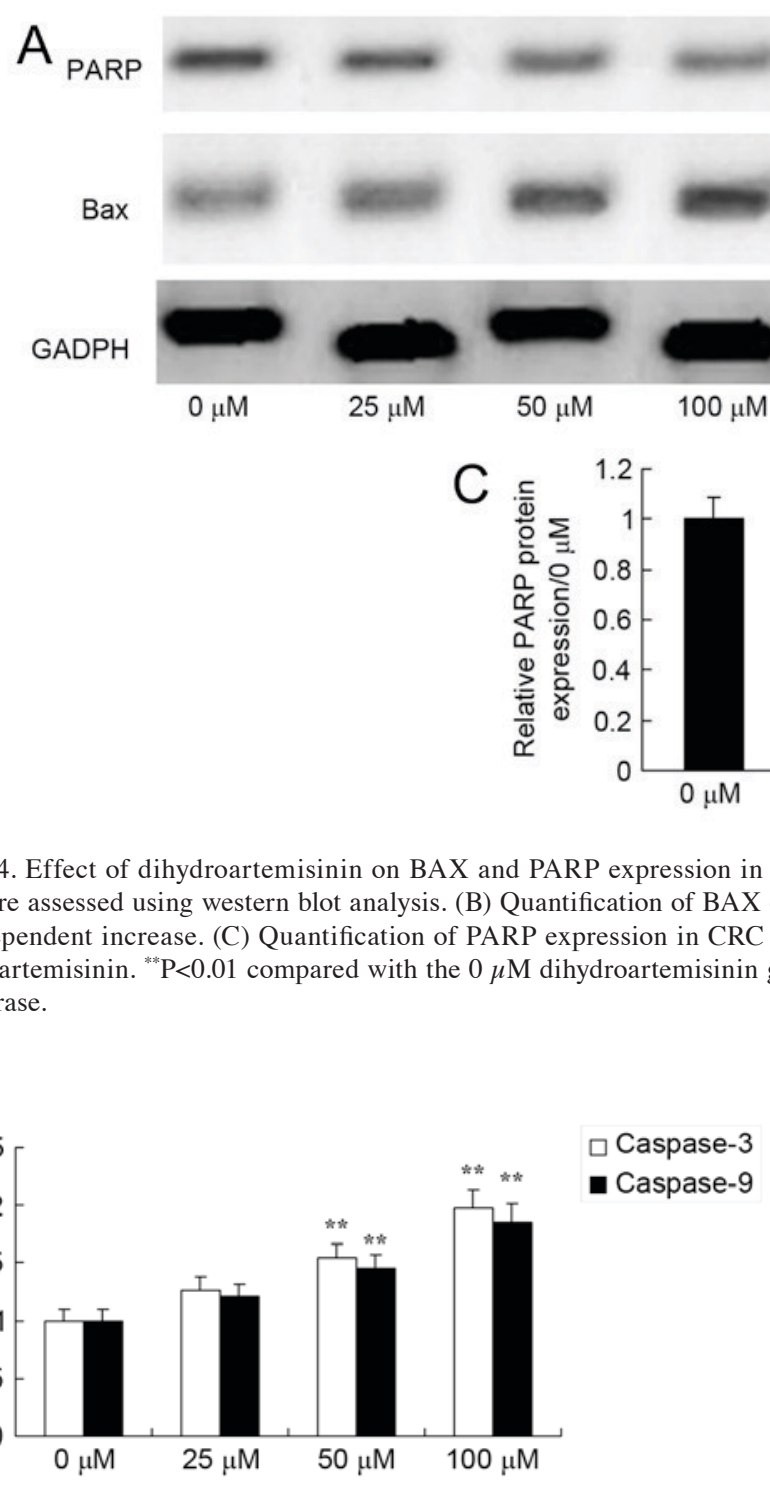

Figure 5. Effect of dihydroartemisinin on caspase-3/9 activity in CRC cells. Treatment with dihydroartemisinin significantly increased caspase- 3 and caspase-9 activity in CRC cells. ${ }^{* *} \mathrm{P}<0.01$ compared with the $0 \mu \mathrm{M}$ dihydroartemisinin group.

transactivated PARP, caspase-3/9 and BAX each serve a role in dihydroartemisinin-induced apoptosis in CRC cells.

The MAPK signaling pathway participates in numerous biological activities of cells with normal physiological function, including proliferation, differentiation, apoptosis and the cell cycle (20). One previous study revealed that this signaling pathway was associated with the biological characteristics of a tumor (20). The ERK/MAPK signaling pathway includes ERK1/2, JNK1/2 and p38 MAPK, and extracellular signals are transmitted inside cells by activating the aforementioned proteins. Thus, inhibiting even one of these molecules may inhibit the entire signal pathway, thereby inhibiting the biological effect of any associated stimulatory molecules $(21,22)$. Therefore, such molecules are expected to be targets for inhibiting the signaling pathway. In the present study, it was revealed that dihydroartemisinin significantly suppressed phosphorylation of ERK1/2 and induced phosphorylation of JNK1/2 and p38 MAPK protein expression in HCT116 cells.
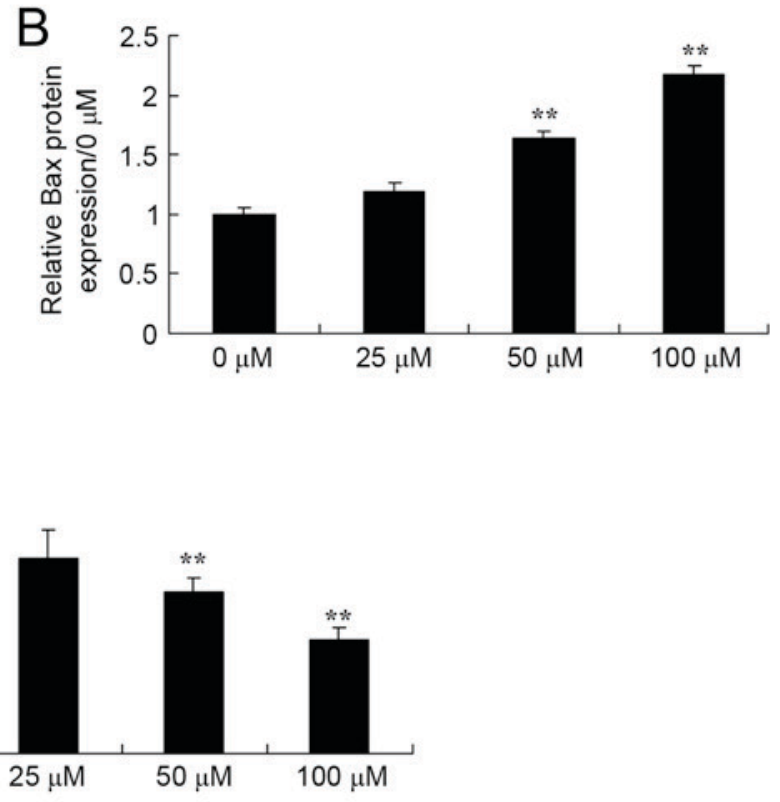

Zhang et al (23) reported that dihydroartemisinin induces apoptosis through the activation of JNK1/2 and p38 MAPK signaling pathways in a human gastric cancer cell line BGC-823. The aforementioned results suggest that ERK1/2, JNK1/2 and p38 MAPK activity are associated with the apoptotic effects of dihydroartemisinin in CRC cells.

STAT3 is a transcription factor which is able to enter the nucleus and bind to DNA fragments of the target gene promoter following activation, in order to regulate the expression of associated genes, resulting in biological effects (24). STAT3 serves an important role in embryonic development and the function of normal cells (9). The abnormal expression and activation of STAT3 was revealed in numerous types of tumor, suggesting that it may promote the development of the tumor $(9,25)$. Previous in vitro experiments identified that STAT3 significantly influenced the invasion of tumor cells (26). The results of the present study revealed that dihydroartemisinin significantly suppressed the phosphorylation of STAT3 in HCT116 cells.

In order to apply these results to the therapeutic treatment of tumors, it is necessary to evaluate a potential association between the activation of STAT3 and disease progression, whether tumors with high phosphorylation level and heterogeneity of the STAT3 tyrosine residue are more sensitive to targeted therapy, the role that other types of STATs serve in the tumor microenvironment, and whether blocking the JAK2/STAT3 signal pathway may activate other signal pathways through a crosstalk effect and thereby result in drug resistance of tumor cells $(9,27)$. With the continuous improvement of in vitro and clinical tests, the JAK2/STAT3 signaling pathway is expected to become a novel target for the treatment of CRC without combination with other drugs, in order to decrease the risk of drug resistance and improve the quality of 
A

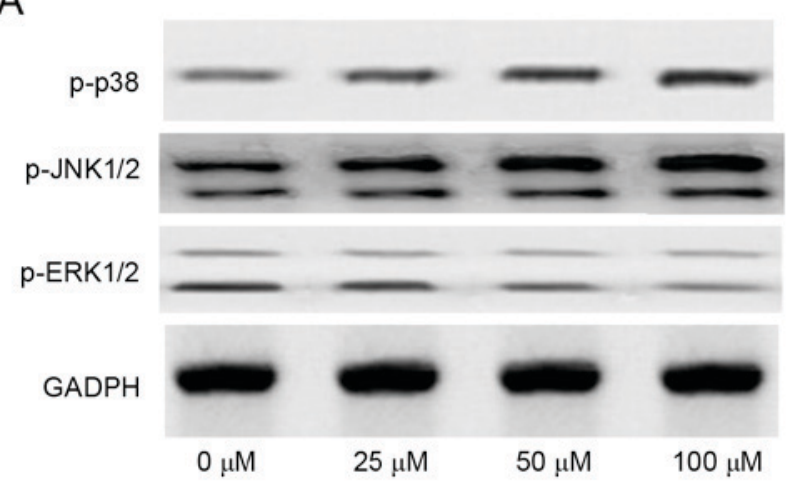

C

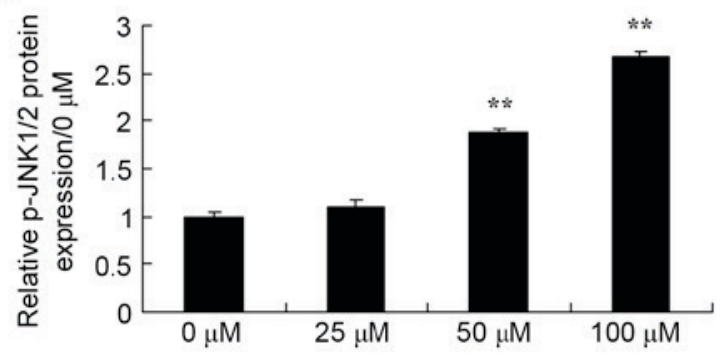

B

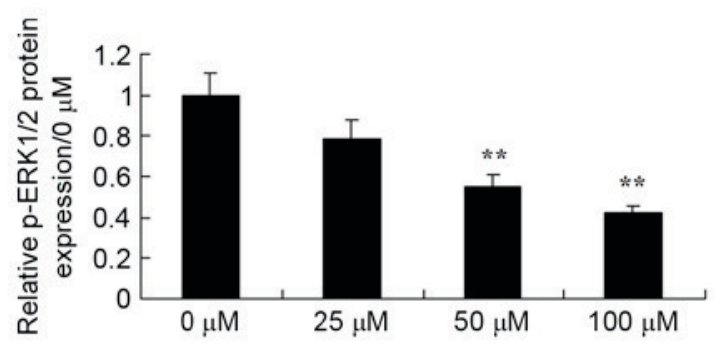

D

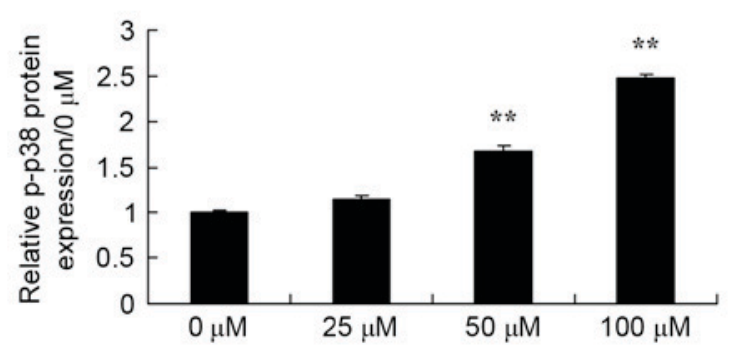

Figure 6. Effect of dihydroartemisinin on phosphorylation of ERK1/2, JNK1/2 and p38 MAPK in CRC cells. (A) The levels of phosphorylated ERK1/2, JNK1/2 and p38 MAPK were assessed using western blotting. (B) The levels of p-ERK1/2 were quantified, revealing a significant dose-dependent decrease following treatment with dihydroartemisinin. (C) The levels of p-JNK1/2 were quantified, revealing a significant dose-dependent increase following treatment with dihydroartemisinin. (D) The levels of p-p38 were quantified, revealing a significant dose-dependent increase following treatment with dihydroartemisinin. ${ }^{* *} \mathrm{P}<0.01$ compared with the $0 \mu \mathrm{M}$ dihydroartemisinin group. p-ERK, phosphorylated extracellular-signal-related kinase; p-JNK, phosphorylated c-Jun N-terminal kinase; p-p38, phosphorylated p38 mitogen-activated protein kinase.

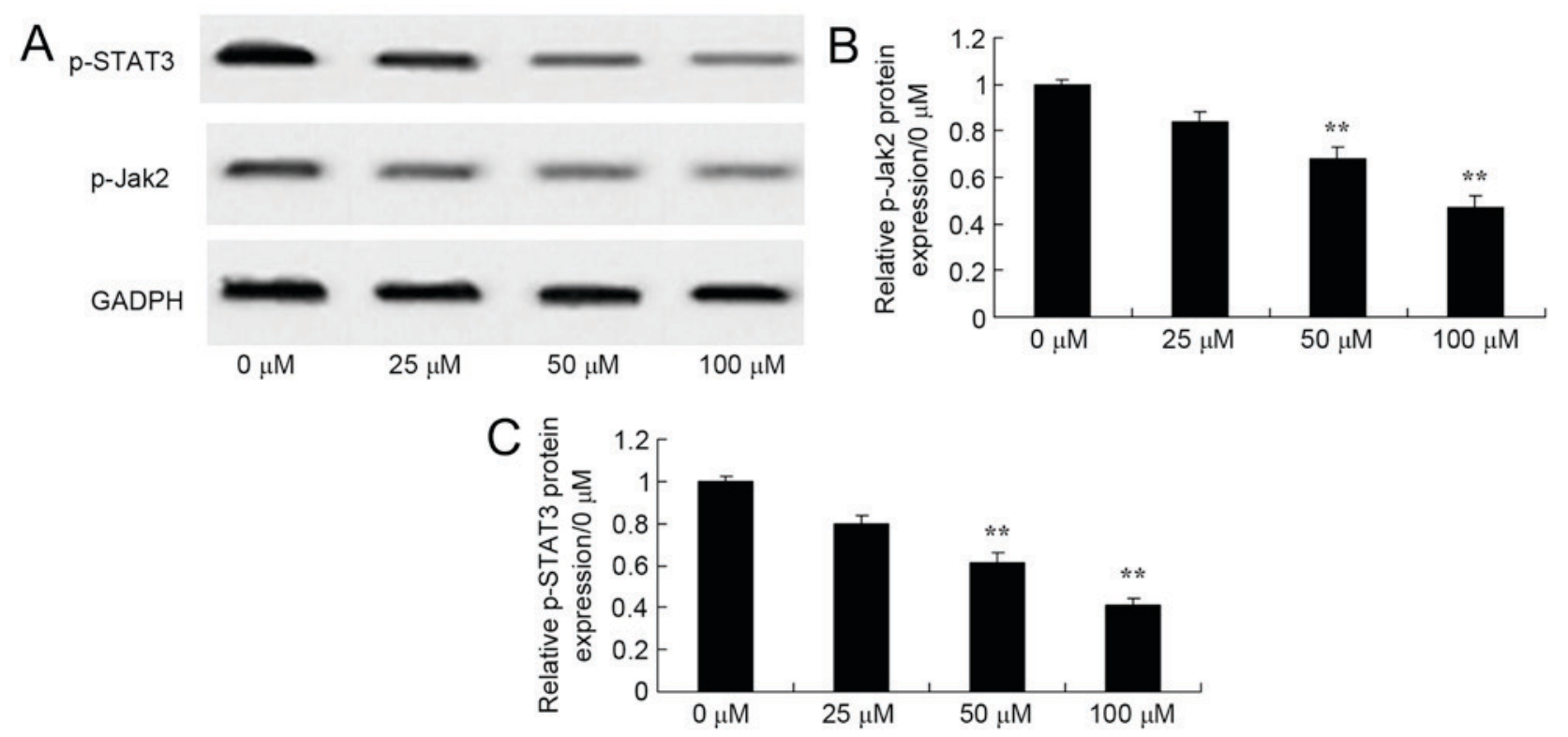

Figure 7. Effect of dihydroartemisinin on phosphorylation of JAK 2 and STAT3 in CRC cells. (A) The effects of dihydroartemisinin on phosphorylation of JAK 2 and STAT3 were assessed using western blot analysis. (B) Quantification of phosphorylation of JAK2 in HCT116 cells revealed a significant dose-dependent decrease following treatment with dihydroartemisinin. (C) Quantification of the phosphorylation of STAT3 in CRC cells revealed a significant dose-dependent decrease following treatment with dihydroartemisinin. ${ }^{* *} \mathrm{P}<0.01$ compared with the $0 \mu \mathrm{M}$ dihydroartemisinin group. p-JAK2, phosphorylated Janus kinase 2 ; p-STAT3, phosphorylated signal transducer and activator of transcription 3.

life of patients (28). Taken together, these results suggest that dihydroartemisinin significantly suppressed the phosphorylation of JAK2 in HCT116 cell. Jia et al (12) demonstrated that dihydroartemisin suppresses squamous cell carcinoma growth through targeting the JAK2/STAT3 signaling pathway. The results of the present study demonstrate the contribution of the JAK2/STAT3 signaling pathway in dihydroartemisinin-induced apoptosis of CRC cells. 
In summary, the present study demonstrated that treatment with dihydroartemisinin decreased cell viability, induced apoptosis, increased caspase-3/9 activities and upregulated BAX protein expression, which is mediated by the PARP/MAPK and JAK2/STAT3 signaling pathways.

\section{References}

1. Grenader T, Nash S, Adams R, Kaplan R, Fisher D, Maughan T and Bridgewater J: Derived neutrophil lymphocyte ratio is predictive of survival from intermittent therapy in advanced colorectal cancer: A post hoc analysis of the MRC COIN study. Br J Cancer 114: 612-615, 2016.

2. Neubauer K, Misa IB, Diakowska D, Kapturkiewicz B, Gamian A and Krzystek-Korpacka M: Nampt/PBEF/visfatin upregulation in colorectal tumors, mirrored in normal tissue and whole blood of colorectal cancer patients, is associated with metastasis, hypoxia, IL1 $\beta$ and anemia. Biomed Res Int 2015: 523930, 2015.

3. Sadahiro S, Morita S, Sasaki K, Sakamoto K, Ohge H, Takahashi T, Tsuchiya T, Sato T, Kondo K, Ogata Y, et al: Treatment rationale and study design for clinical trial on the efficacy of UFT/LV for stage II colorectal cancer with risk factors for recurrence (JFMC46-1201). Clin Colorectal Cancer 14 277-280, 2015.

4. Ulivi P, Scarpi E, Passardi A, Marisi G, Calistri D, Zoli W, Del Re M, Frassineti GL, Tassinari D, Tamberi S, et al: eNOS polymorphisms as predictors of efficacy of bevacizumab-based chemotherapy in metastatic colorectal cancer: Data from a randomized clinical trial. J Transl Med 13: 258, 2015.

5. Iwasa S, Nagashima K, Yamaguchi T, Matsumoto H, Ichikawa Y, Goto A, Yasui H, Kato K, Okita NT, Shimada Y and Yamada Y: S-1 and irinotecan with or without bevacizumab versus 5 -fluorouracil and leucovorin plus oxaliplatin with or without bevacizumab in metastatic colorectal cancer: A pooled analysis of four phase II studies. Cancer Chemother Pharmacol 76: 605-614,2015.

6. Monnien F, Zaki H, Borg C, Mougin C, Bosset JF, Mercier M, Arbez-Gindre F and Kantelip B: Prognostic value of phosphorylated STAT3 in advanced rectal cancer: A study from 104 French patients included in the EORTC 22921 trial. J Clin Pathol 63: 873-878, 2010.

7. Han S, Jeong AJ, Yang H, Bin Kang K, Lee H, Yi EH, Kim BH, Cho CH, Chung JW, Sung SH and Ye SK: Ginsenoside 20(S)-Rh2 exerts anti-cancer activity through targeting IL-6-induced JAK2/STAT3 pathway in human colorectal cancer cells. J Ethnopharmacol 194: 83-90, 2016.

8. van't Sant HP, Kamman A, Hop WC, van der Heijden M, Lange JF and Contant CM: The influence of mechanical bowel preparation on long-term survival in patients surgically treated for colorectal cancer. Am J Surg 210: 106-110, 2015.

9. Ren W, Shen S, Sun Z, Shu P, Shen X, Bu C, Ai F, Zhang X, Tang A, Tian L, et al: Jak-STAT3 pathway triggers DICER1 for proteasomal degradation by ubiquitin ligase complex of CUL4A(DCAF1) to promote colon cancer development. Cancer Lett 375: 209-220, 2016.

10. Lin R, Zhang Z, Chen L, Zhou Y, Zou P, Feng C, Wang L and Liang G: Dihydroartemisinin (DHA) induces ferroptosis and causes cell cycle arrest in head and neck carcinoma cells. Cancer Lett 381: 165-175, 2016

11. Li Y, Wang Y, Kong R, Xue D, Pan S, Chen H and Sun B: Dihydroartemisinin suppresses pancreatic cancer cells via a microRNA-mRNA regulatory network. Oncotarget 7: 62460-62473, 2016.

12. Jia L, Song Q, Zhou C, Li X, Pi L, Ma X, Li H, Lu X and Shen Y: Dihydroartemisinin as a putative STAT3 Inhibitor, suppresses the growth of head and neck squamous cell carcinoma by targeting Jak2/STAT3 signaling. PLoS One 11: e0147157, 2016.

13. Chen Q, Chen L, Kong D, Shao J, Wu L and Zheng S: Dihydroartemisinin alleviates bile duct ligation-induced liver fibrosis and hepatic stellate cell activation by interfering with the PDGF- $\beta R / E R K$ signaling pathway. Int Immunopharmacol 34 250-258, 2016.
14. Fizazi K, Faivre L, Lesaunier F, Delva R, Gravis G, Rolland F, Priou F, Ferrero JM, Houede N, Mourey L, et al: Androgen deprivation therapy plus docetaxel and estramustine versus androgen deprivation therapy alone for high-risk localised prostate cancer (GETUG 12): A phase 3 randomised controlled trial. Lancet Oncol 16: 787-794, 2015.

15. Williams GR, Nyrop KA, Deal AM, Muss HB and Sanoff HK: Self-directed physical activity intervention in older adults undergoing adjuvant chemotherapy for colorectal cancer: Design of a randomized controlled trial. Contemp Clin Trials 42: 90-97, 2015.

16. Tentori L, Muzi A, Dorio AS, Dolci S, Campolo F, Vernole P, Lacal PM, Praz F and Graziani G: MSH3 expression does not influence the sensitivity of colon cancer HCT116 cell line to oxaliplatin and poly(ADP-ribose) polymerase (PARP) inhibitor as monotherapy or in combination. Cancer Chemother Pharmacol 72: 117-125, 2013.

17. Aredia F, Giansanti V, Mazzini G, Savio M, Ortiz LM, Jaadane I, Zaffaroni N, Forlino A, Torriglia A and Scovassi AI: Multiple effects of the $\mathrm{Na}(+) / \mathrm{H}(+)$ antiporter inhibitor HMA on cancer cells. Apoptosis 18: 1586-1598, 2013.

18. Abu-Sanad A, Wang Y, Hasheminasab F, Panasci J, Noë A, Rosca L, Davidson D, Amrein L, Sharif-Askari B, Aloyz R and Panasci L: Simultaneous inhibition of ATR and PARP sensitizes colon cancer cell lines to irinotecan. Front Pharmacol 6: 147, 2015.

19. Wang Z, Hu W, Zhang JL, Wu XH and Zhou HJ: Dihydroartemisinin induces autophagy and inhibits the growth of iron-loaded human myeloid leukemia K562 cells via ROS toxicity. FEBS Open Bio 2: 103-112, 2012.

20. Lin J, Li Q, Chen H, Lin H, Lai Z and Peng J: Hedyotis diffusa Willd. Extract suppresses proliferation and induces apoptosis via IL-6-inducible STAT3 pathway inactivation in human colorectal cancer cells. Oncol Lett 9: 1962-1970, 2015.

21. Gupta J, Igea A, Papaioannou M, Lopez-Casas PP, Llonch E, Hidalgo M, Gorgoulis VG and Nebreda AR: Pharmacological inhibition of p38 MAPK reduces tumor growth in patient-derived xenografts from colon tumors. Oncotarget 6: 8539-8551, 2015.

22. Zhuang Q, Hong F, Shen A, Zheng L, Zeng J, Lin W, Chen Y, Sferra TJ, Hong Z and Peng J: Pien Tze Huang inhibits tumor cell proliferation and promotes apoptosis via suppressing the STAT3 pathway in a colorectal cancer mouse model. Int J Oncol 40: 1569-1574, 2012.

23. Zhang S, Shi L, Ma H, Li H, Li Y, Lu Y, Wang Q and Li W: Dihydroartemisinin induces apoptosis in human gastric cancer cell line BGC-823 through activation of JNK1/2 and p38 MAPK signaling pathways. J Recept Signal Transduct Res 17: 174-180, 2017.

24. Sanchez-Lopez E, Flashner-Abramson E, Shalapour S, Zhong Z, Taniguchi K, Levitzki A and Karin M: Targeting colorectal cancer via its microenvironment by inhibiting IGF-1 receptor-insulin receptor substrate and STAT3 signaling. Oncogene 35: 2634-2644, 2016.

25. You L, Wang Z, Li H, Shou J, Jing Z, Xie J, Sui X, Pan H and Han W: The role of STAT3 in autophagy. Autophagy 11: 729-739, 2015.

26. Yang H, Yamazaki T, Pietrocola F, Zhou H, Zitvogel L, Ma Y and Kroemer G: STAT3 inhibition enhances the therapeutic efficacy of immunogenic chemotherapy by stimulating type 1 interferon production by cancer cells. Cancer Res 75: 3812-3822, 2015.

27. Yu H, Yue X, Zhao Y, Li X, Wu L, Zhang C, Liu Z, Lin K, $\mathrm{Xu}$-Monette $\mathrm{ZY}$, Young $\mathrm{KH}$, et al: LIF negatively regulates tumour-suppressor p53 through Stat3/ID1/MDM2 in colorectal cancers. Nat Commun 5: 5218, 2014.

28. Xiong H, Du W, Zhang YJ, Hong J, Su WY, Tang JT, Wang YC, Lu R and Fang JY: Trichostatin A, a histone deacetylase inhibitor, suppresses JAK2/STAT3 signaling via inducing the promoter-associated histone acetylation of SOCS1 and SOCS3 in human colorectal cancer cells. Mol Carcinog 51: 174-184, 2012. 